\title{
Fit für den Facharzt INTENSIV - TECHNIK geht in die zweite Runde
}

Am 3. und 4. September findet zum zweiten Mal der „Fit für den Facharzt INTENSIV

- TECHNIK“-Kurs unter der wissenschaftlichen Leitung von Dr. rer. nat. Kerstin Jungnickel als Onlineveranstaltung statt.

Der TECHNIK Kurs dient als Ergänzung zum „FIT für den Facharzt INTENSIV“-Kurs und erläutert die physikalisch-technisch relevanten Themen für die Facharztprüfung, wozu insbesondere die Gerätetechnik und Bildentstehung beim Röntgen, CT und MRT gehören.

Physik und Technik aller radiologischen Geräte werden Ihnen von erfahrenen Medizinphysikexpertinnen und Medizinphysikexper- ten entsprechend des neuen WeiterbildungsCurriculums zusammenfassend dargestellt.

Unter anderem erwarten Sie folgende Themen sowie Referentinnen und Referenten:

- Strahlenphysik (Ionisierende Strahlung, Wechselwirkung mit Materie) J. Ammon (Nürnberg)

- Durchleuchtung/Angiographie (DSA, Kontrastmittel, Strahlenschutz des Personals)

B. Leonhäuser (Berlin)

- CT (Gerätetechnik, Bildentstehung, HU, Protokolle, Kontrastmittel, Bildqualität und Dosis) W. Stiller (Heidelberg)
- MRT I (Gerätetechnik, Bildentstehung, Sequenzen I)

B. Keil (Gießen)

- IT (PACS, RIS, DMS)

C. Piel (Köln)

Alle Informationen, das Programm sowie den Anmeldelink finden Sie im Veranstaltungskalender der Akademie für Fort- und Weiterbildung unter www.drgakademie.de $>$ Veranstaltungskalender $>3$. September Fit für den Facharzt INTENSIV - TECHNIK 